\title{
Síndrome compartimental agudo del miembro inferior: estado actual
}

\author{
Acute compartment syndrome of the lower extremity: An update
}

\author{
David Felipe Muñoz-Pérez ${ }^{\mathbb{D}}$, Rolando Medina-Rojas² $\mathbb{D}$, Juan Felipe Sanjuan-Marín ${ }^{3} \mathbb{D}$, \\ Roberto José Rodríguez-Flórez ${ }^{4} \mathbb{D}$, Nelson Daniel Sánchez-Arroyo ${ }^{5} \mathbb{D}$
}

1 Médico, residente de Cirugía general, Universidad Surcolombiana, Neiva, Colombia.

2 Médico, especialista en Cirugía general y Cirugía de Trauma; jefe, Servicio de Cirugía general, Hospital Universitario Hernando Moncaleano Perdomo; coordinador, posgrado de Cirugía general, Universidad Surcolombiana, Neiva, Colombia.

3 Médico, especialista en Cirugía general; profesor catedrático, posgrado de Cirugía general, Universidad Surcolombiana, Neiva, Colombia.

4 Médico, especialista en Cirugía general, fellow Cirugía de colon y recto, Universidad Militar Nueva Granada, Bogotá, D.C., Colombia.

5 Médico, Universidad Surcolombiana, Neiva, Colombia.

\section{Resumen}

El síndrome compartimental agudo del miembro inferior es una urgencia médica, que puede conllevar una importante morbilidad para el paciente y que puede tener implicaciones medicolegales para el personal médico. Afecta cerca de 3,1 por 100.000 habitantes en el mundo occidental, con predominio de hombres y jóvenes. Debido a una alteración de la perfusión tisular, por la disposición de los músculos de las extremidades en compartimientos compactos, con poca tolerancia a variaciones en la presión, puede causar isquemia, con cambios irreversibles a nivel muscular y nervioso, falla multiorgánica y la muerte, de no tratarse oportunamente.

El trauma de la extremidad inferior asociado a fractura es la principal causa del síndrome compartimental agudo. El diagnóstico puede realizarse por evaluación clínica, mediante las clásicas seis "P" de la isquemia, o de manera objetiva, al medir la presión intracompartimental con dispositivos especializados. La fasciotomía descompresiva es un procedimiento quirúrgico mediante el cual se inciden las fascias de los compartimientos musculares, permitiendo la disminución de las presiones a ese nivel, y se constituye en el único tratamiento eficaz para el síndrome compartimental agudo. Las complicaciones posquirúrgicas no son infrecuentes, siendo la perdida de la extremidad la más grave de todas. Un correcto conocimiento y aplicación de la técnica quirúrgica evitará procedimientos inadecuados, que impliquen mayor riesgo de resultados adversos.

Presentamos una revisión de los aspectos fundamentales de esta patología potencialmente catastrófica.

Palabras clave: síndromes compartimentales; isquemia; fasciotomía; amputación; extremidad inferior.

Fecha de recibido: 16/05/2020 - Fecha de aceptación: 25/08/2020

Correspondencia: David Felipe Muñoz, Universidad Surcolombiana, Calle 9 \# 15-25, Neiva, Colombia.

Teléfono: (098) 8715907 ext. 4266. Correo electrónico pipetrored@gmail.com

Citar como: Muñoz-Pérez DF, Medina-Rojas R, Sanjuan-Marín JF, Rodríguez-Flórez RJ, Sánchez-Arroyo ND. Síndrome compartimental agudo del miembro inferior: estado actual. Rev Colomb Cir. 2021;36:132-43. https://doi.org/10.30944/20117582.663

Este es un artículo de acceso abierto bajo una Licencia Creative Commons - BY-NC-ND https://creativecommons.org/licenses/by-nc$\mathrm{nd} / 4.0 /$ deed.es 


\begin{abstract}
Acute compartment syndrome of the lower limb is a medical emergency, which can entail significant morbidity for the patient and which may have medico-legal implications for medical personnel. It affects about 3.1 per 100,000 inhabitants in the Western world, with a predominance of men and young people. Ischemia can be caused after an alteration of tissue perfusion, due to the arrangement of the muscles of the extremities in compact compartments, with little tolerance to variations in pressure, with irreversible changes at the muscular and nervous level, and multiorgan failure and death if not treated promptly.

Trauma to the lower extremity associated with fracture is the main cause of acute compartment syndrome. Diagnosis can be made by clinical evaluation, using the classic six "P" of ischemia, or objectively by measuring intracompartmental pressure with specialized devices. Decompressive fasciotomy is a surgical procedure by which the fasciae of the muscle compartments are incised, allowing the pressure to be reduced at that level, and it is the only effective treatment for acute compartment syndrome. Postoperative complications are not uncommon, limb loss being the most serious of all. A correct knowledge and application of the surgical technique will avoid inappropriate procedures, which imply a greater risk of adverse results.
\end{abstract}

We present a review of the fundamental aspects of this potentially catastrophic pathology.

Keywords: compartment syndromes; ischemia; fasciotomy; amputation; lower extremity.

\section{Introducción}

El síndrome compartimental agudo (SCA) del miembro inferior tiene el potencial de causar morbilidad devastadora en los pacientes y riesgos legales para los médicos involucrados en su tratamiento ${ }^{1-3}$. Se caracteriza por una presión aumentada en uno o más compartimientos musculares de la extremidad, asociado a una perfusión celular afectada y disminuida ${ }^{4}$. Los datos epidemiológicos son invaluables para identificar pacientes con alto riesgo de desarrollar la enfermedad, quienes por ende se benefician de una vigilancia y monitoreo estricto de la presión intracompartimental.

La incidencia del SCA en el mundo occidental se calcula en 3,1 por 100.000 habitantes. Los hombres son afectados más frecuentemente que las mujeres, con una relación de 10:1, al igual que los pacientes jóvenes, siendo la edad de presentación promedio de 32 años para la población general, de 30 años para los hombres y 44 años para las mujeres ${ }^{4-6}$.

A pesar de la relativa baja incidencia, es fundamental para el personal asistencial un adecuado dominio del tema, ya que el reconocimiento temprano constituye la principal estrategia para la prevención de sus complicaciones ${ }^{7,8}$. Las secuelas del síndrome compartimental que no recibe tratamiento fueron originalmente publicadas por Volkmann en $1881^{9}$. Su artículo describe la aparición de isquemia grave en extremidades traumatizadas, que evolucionaba rápidamente a contractura y parálisis. En ese momento la teoría apuntaba como principal causa al uso de vendajes excesivamente ajustados en el manejo de heridas ${ }^{10}$. La enfermedad fue descrita en mayor detalle en una serie de casos reportados durante la segunda guerra mundial, en donde los autores identificaron lesiones en extremidades que ocasionaban edema significativo y evolucionaban a pulsos disminuidos o ausentes en el miembro afectado, choque sistémico, gangrena y falla renal progresiva que llevaba a la muerte ${ }^{11}$. En 1949, Carter et al lograron caracterizar de manera más especifica el cuadro clínico, y estipularon que estaba ocasionado principalmente por traumatismo muscular, con un incremento subsecuente de la presión intracompartimental, que impedía el flujo sanguíneo adecuado y culminaba en necrosis muscular y complicaciones potencialmente letales ${ }^{10}$.

El síndrome compartimental puede aparecer en cualquier región anatómica en donde los músculos se distribuyan en compartimientos (brazo, an- 
tebrazo, mano, región glútea y músculos lumbares paraespinales). Aunque generalmente ocurre de forma aguda, también puede presentarse de forma subaguda, y en ocasiones crónica ${ }^{7}$. El objetivo de este artículo era revisar el tema y presentar los aspectos relevantes de la anatomía, fisiología, diagnostico, manejo y complicaciones del SCA del miembro inferior.

\section{Anatomía y fisiopatología}

Los músculos de las extremidades se encuentran distribuidos en compartimientos compactos, delimitados por fascias, septos de tejido conectivo y estructuras óseas. Esos compartimientos constituyen un sistema cerrado, con poca tolerancia al aumento de presión, por los que atraviesan los vasos sanguíneos, linfáticos y nervios de los miembros. Los compartimientos con menor permisividad o capacidad de dilatarse son aquellos más susceptibles a desarrollar el SCA.

La pierna y sus 4 compartimientos, anterior, lateral, posterior superficial y posterior profundo (figura 1), representan la región anatómica más afectada por el síndrome, siendo el compartimiento anterior el más frecuentemente comprometido ${ }^{3,12}$. Este compartimiento está delimitado por la tibia y el peroné, la membrana interósea y el septo intermuscular anterior, y contiene los principales

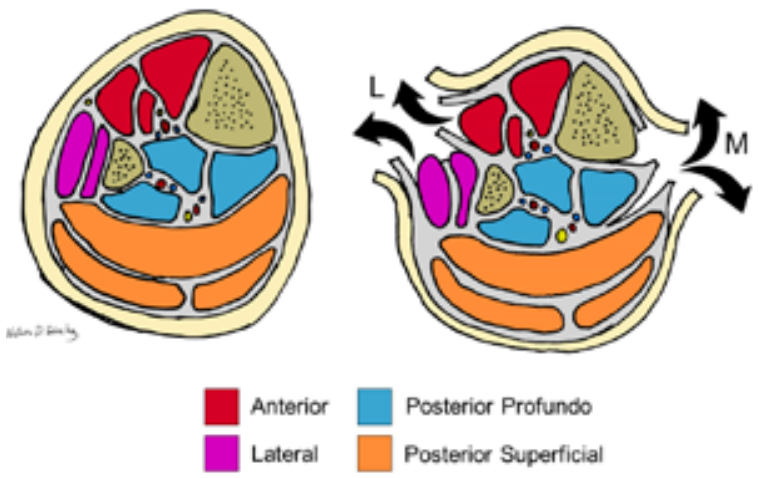

Figura 1. Compartimientos de la pierna y sus abordajes en fasciotomía: Se ilustran los cuatro compartimientos del miembro inferior. El abordaje medial permite liberar los compartimientos posterior y posterior profundo. El abordaje lateral permite liberar los compartimientos anterior y lateral. músculos extensores del pie ${ }^{1}$. El SCA en el muslo tiene una incidencia baja. A este nivel existen 3 compartimientos musculares, anterior, posterior y medial (figura 2), y al igual que en la pierna, el anterior es el más afectado ${ }^{3}$. El pie es una estructura compleja, en el cual se describen 9 compartimientos musculares, sin que haya un consenso generalizado respecto a estos ${ }^{13}$. Los compartimientos del muslo y la pierna, y sus contenidos, se resumen en la tabla 1.

Se han descrito múltiples teorías que explican la fisiopatología del síndrome compartimental agudo, las cuales tienen en común la anoxia celular, como resultado de una serie de procesos fisiológicos complejos e interconectados. Matsen y Krugmire ${ }^{14}$ describieron la teoría del gradiente arterio-venoso capilar, que es la explicación más ampliamente acogida actualmente (figura 3). Esta considera que la presión intracompartimental normal se ubica alrededor de los $10 \mathrm{~mm} / \mathrm{Hg}$ y sugiere que un aumento significativo de la misma, causada bien sea por un incremento del volumen intracompartimental (por ejemplo, hemorragia o edema) o por disminución del volumen del compartimiento (por ejemplo, vendajes o férulas excesivamente ajustadas), ocasiona aumento de la presión venosa intraluminal y altera a nivel capilar el gradiente de presión arterio-venoso normal,

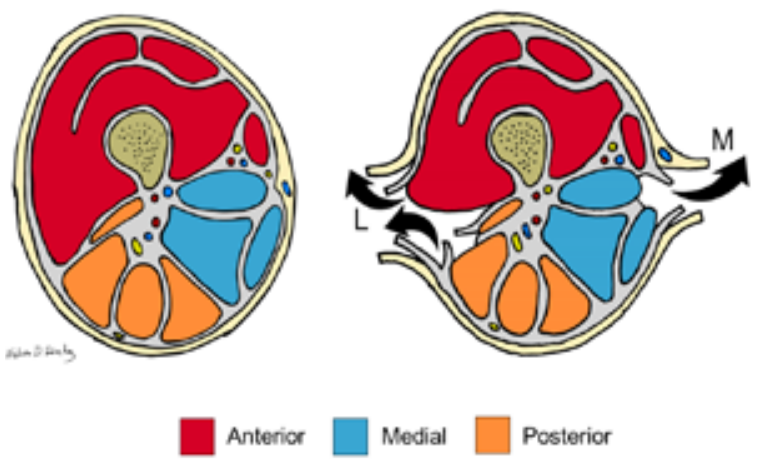

Figura 2. Compartimientos del muslo y sus abordajes en fasciotomía: Se ilustran los tres compartimientos del muslo. El abordaje lateral único se prefiere para la liberación de todos los compartimientos, pero puede utilizarse en combinación con un abordaje medial para liberar el compartimiento medial con menor dificultad técnica. 
Tabla 1. Compartimientos del miembro inferior y sus principales estructuras.

\begin{tabular}{ll}
\hline $\begin{array}{l}\text { Localización } \\
\text { anatómica }\end{array}$ & \multicolumn{1}{c}{ Compartimiento } \\
\hline & Anterior: \\
& -músculos tibial anterior, extensor largo de \\
& los dedos y extensor largo del hallux \\
& -arteria y vena tibial anterior \\
& -nervio peroneo profundo \\
\cline { 2 - 2 } & Lateral: \\
& -músculos peroneo largo y peroneo corto \\
& -nervio peroneo superficial \\
\hline Pierna & Posterior Superficial: \\
& -músculos gastrocnemio y sóleo \\
& Posterior Profundo: \\
& -músculos flexor largo de los dedos, flexor \\
& largo del hallux y poplíteo \\
& -arteria y vena tibial posterior \\
& -nervio tibial \\
\hline & Anterior: \\
& -músculos vasto medio, vasto intermedio, \\
& vasto lateral, recto femoral y sartorio \\
& -arteria y vena femoral común y superficial \\
& -nervio femoral \\
& Posterior: \\
& -músculos bíceps femoral, semitendinoso, \\
& semimembranoso \\
& -nervio ciático \\
& -arteria y vena femoral profunda \\
& Medial: \\
& -músculos aductor largo, aductor corto, \\
& aductor magno y grácil \\
\hline Muslo &
\end{tabular}

afectando el flujo sanguíneo, que usualmente discurre en la microcirculación desde las arteriolas hacia las vénulas y de mayor a menor presión. Lo anterior tiene como implicaciones principales la disminución en el aporte tisular de sangre arterial oxigenada y la imposibilidad del drenaje de la sangre venosa desoxigenada ${ }^{14-16}$.

La elevación subsecuente de la presión hidrostática ocasiona aumento en la permeabilidad capilar, fuga del plasma hacia el intersticio y edema tisular, lo que a su vez contribuye a un aumento sostenido de la presión compartimental. Esta suerte de circulo vicioso en el que la presión se eleva a medida que el flujo capilar se deteriora progresivamente, termina por colapsar los vasos

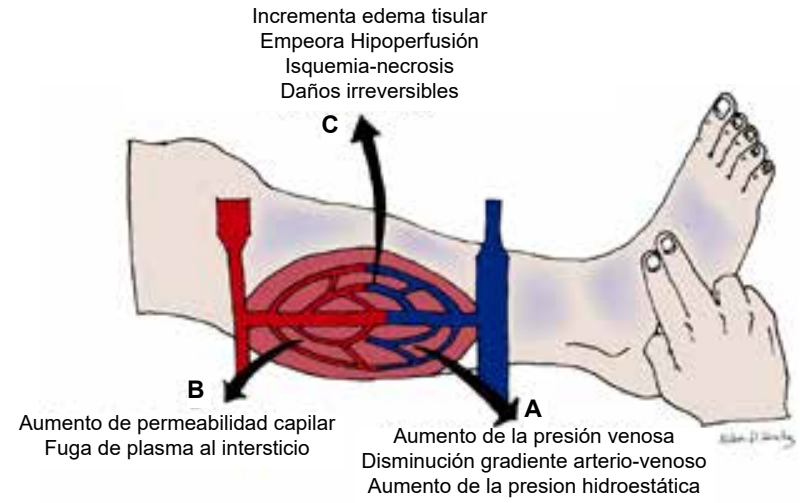

Figura 3. Principales eventos fisiopatológicos en el síndrome compartimental agudo: un aumento significativo de las presiones intracompartimentales ocasionan colapso venoso y alteración del gradiente arterio-venoso fisiológico con aumento subsecuente de la presión hidrostática capilar (A). Esto a su vez eleva la permeabilidad arterial, con subsecuente fuga plasmática al intersticio (B), lo cual termina por incrementar sostenidamente la presión intracompartimental hasta ocasionar isquemia, necrosis y daños irreversibles.

linfáticos y finalmente compromete la irrigación arterial, lo cual culmina en anoxia celular y necrosis irreversible ${ }^{14,15}$. La presión intracompartimental capaz de comprometer la perfusión tisular normal se encuentran cercana a la presión diastólica, entre 10 a $30 \mathrm{~mm} / \mathrm{Hg}$, y la oxigenación se afecta a medida que estas se acercan o superan la presión arterial media.

Por lo tanto, el desarrollo del SCA de la extremidad depende no solo de la presión del compartimiento muscular, sino también de la presión sanguínea sistémica y, debido a esto, los pacientes hipotensos, como los politraumatizados, se encuentran en mayor riesgo de presentarlo ${ }^{14}$.

Los periodos más prolongados de isquemia implican mayor morbimortalidad para el paciente. Las alteraciones nerviosas reversibles se presentan a partir de 30 minutos luego de la instauración del SCA y las musculares entre 2-4 horas, mientras que los cambios irreversibles aparecen a nivel muscular desde las 6 horas y a nivel nervioso a partir de las 12 horas. Este corto periodo, es el que impone el desafío diagnostico para los médicos tratantes, quienes deben tener como meta, identificar los pacientes con el SCA y el consecuente riesgo de presentar cambios irreversibles ${ }^{16}$. 


\section{Etiología y factores de riesgo}

Las causas del SCA del miembro inferior son variadas y se resumen en la tabla 2 . Como se mencionó previamente, los hombres se ven afectados en mayor medida que las mujeres y los individuos jóvenes son más propensos al desarrollo de SCA del miembro inferior ${ }^{4,17}$. De hecho, ser joven ha demostrado ser el factor de riesgo más importante para el desarrollo del síndrome, el cual tiene prevalencia tres veces más alta en pacientes menores de 35 años, posiblemente en relación a la mayor densidad muscular y compartimientos musculares compactos propios de esta población ${ }^{18}$. Los individuos de sexo masculino además suelen verse involucrados en un mayor número de profesiones de riesgo y lesiones por traumatismos de distinta índole ${ }^{3}$. Los pacientes mayores tienden a presentar distintos grados de sarcopenia y una perfusión tisular aumentada por presencia de hipertensión sistémica, lo cual puede explicar el factor protector con respecto al SCA en este grupo etario ${ }^{4}$.

El trauma de la extremidad es el principal factor etiológico, en particular cuando se asocia a fracturas, las cuales están presentes en el $69 \%$ de todos los casos de SCA, siendo la fractura tibial la más prevalente $(30 \%)^{4,19}$. La lesión de tejidos blandos es la segunda etiología en frecuencia (23\%), sin que se

Tabla 2. Causas comunes del síndrome compartimental agudo del miembro inferior

\begin{tabular}{|c|c|}
\hline Ortopédicas & $\begin{array}{l}\text { Fractura tibial } \\
\text { Fractura femoral } \\
\text { Artroplastia total de rodilla }\end{array}$ \\
\hline Vasculares & $\begin{array}{l}\text { Lesiones arteriales y/o venosas } \\
\text { Procedimientos de revascularización } \\
\text { Trombosis venosa profunda } \\
\text { Hemofilia (hematoma intramuscular) }\end{array}$ \\
\hline Tejidos Blandos & $\begin{array}{l}\text { Aplastamiento sin fractura } \\
\text { Quemaduras } \\
\text { Contusión muscular con edema } \\
\text { Mordedura de serpiente } \\
\text { Miositis viral }\end{array}$ \\
\hline latrogénicas & $\begin{array}{l}\text { Torniquete } \\
\text { Anticoagulación } \\
\text { Férulas o vendajes compresivos } \\
\text { Extravasación de medicamentos } \\
\text { Posición de litotomía prolongada }\end{array}$ \\
\hline
\end{tabular}

pueda identificar un antecedente de traumatismo claro en muchos casos ${ }^{3,4,20}$. Entre los factores que pueden predisponer al desarrollo del síndrome se incluyen las lesiones por aplastamiento, el uso de anticoagulantes, la sobredosis en pacientes farmacodependientes y la presencia de comorbilidades ${ }^{17}$. Adicionalmente, se han descrito casos de SCA en extremidades no lesionadas, por una respuesta inflamatoria sistémica severa con fuga capilar o por una reanimación hídrica agresiva en el manejo de sepsis y otras patologías ${ }^{21}$. Pese a la percepción generalizada de que las lesiones de alta energía y las fracturas abiertas de alto grado en la clasificación de Gustilo y Anderson se asocian mayormente al SCA, se ha demostrado que la incidencia de la patología también es significativa en fracturas cerradas ${ }^{22}$.

En la extremidad traumatizada, múltiples factores juegan un rol importante en el desarrollo de la patología, incluyendo la presencia de lesiones vasculares, la extensión del trauma de tejidos blandos y la hipotensión sistémica ${ }^{23}$. En cuanto al origen traumático, Branco et al. condujeron el mayor estudio, hasta la fecha, para evaluar la incidencia y la caracterización de los pacientes llevados a fasciotomía de miembro inferior por SCA, en el cual incluyeron todos los pacientes que sufrieron traumatismo de extremidades en un periodo de 10 años, para un total de 10.135 pacientes, de los cuales 288 requirieron fasciotomía ${ }^{3}$. Se encontró que la incidencia varió significativamente de acuerdo con el mecanismo del trauma, desde $0,9 \%$ en accidente de tránsito hasta 8,6 \% en herida por proyectil de arma de fuego. El tipo de trauma también jugó un papel importante, de los pacientes que requirieron fasciotomía descompresiva el 2,2 \% presentaban fracturas cerradas, en comparación con el 41,8 \% que sufrieron trauma vascular y venoso combinado. Además, una regresión logística permitió identificar que, en el contexto de trauma de extremidad, la presencia de lesión vascular, requerimiento de trasfusión de glóbulos rojos, sexo masculino, fractura abierta, herida por proyectil de arma de fuego, un ISS (Injury Severity Score, por sus siglas en inglés) mayor de 16 y edad menor de 55 años, fueron predicto- 
res independientes de desarrollo de SCA y necesidad de fasciotomía. Otros autores han descrito también una mayor incidencia de la patología en lesiones con trauma arteriovenoso combinado, fractura asociada a trauma vascular y luxación asociada a trauma vascular ${ }^{22}$.

\section{Diagnóstico}

Un retraso en el diagnóstico del síndrome compartimental puede tener consecuencias graves, por lo que ante sospecha de la enfermedad se necesita una respuesta rápida por parte de los tratantes. El diagnóstico puede realizarse de manera clínica o mediante medición objetiva de las presiones intracompartimentales por medio de dispositivos especializados ${ }^{8}$.

Los signos cardinales del SCA de la extremidad incluyen las llamadas seis " $P$ " de la isquemia: dolor (pain), parestesia, poiquilotermia, palidez, parálisis y ausencia o pérdida del pulso ${ }^{4}$. Se ha demostrado que algunos pacientes con síndrome compartimental no presentan dolor, por lo que la ausencia del mismo no es sinónimo de ausencia de enfermedad ${ }^{24}$. La presencia del dolor como único síntoma parece tener mejor rendimiento diagnóstico, especialmente cuando tiene las siguientes características: ser desproporcionado con los hallazgos físicos, presentarse con el estiramiento pasivo de los músculos de los compartimientos involucrados, aparecer con la palpación del compartimiento involucrado y asociarse a tumefacción del compartimiento ${ }^{4}$.

Un estudio llevado a cabo por Ulmer et al. calculó el valor predictivo de estas manifestaciones cardinales, demostrando que todos ellos fueron menos sensibles (promedio $16 \%$ ) que específicos (promedio $97 \%$ ), con valores predictivos positivos (VPP) que variaron del 11 al $15 \%$ y valores predictivos negativos (VPN) de $98 \%$. Lo anterior sugiere que, en ausencia de síntomas cardinales es menos probable que el paciente tenga SCA del miembro inferior. Además, la baja sensibilidad y bajo VPP, implica que un signo clínico por sí solo es un pobre predictor de enfermedad, lo que cambia si se toman en conjunto, ya que se comprobó que la sensibilidad para tres y cuatro manifestaciones presentes al mismo tiempo fue de 93 y $98 \%$, respectivamente ${ }^{25}$.

En los centros donde de rutina se mide la presión intracompartimental, la decisión de realizar fasciotomía comúnmente se basa en este resultado. Esta herramienta es de suma utilidad en los pacientes cuya condición clínica no permite un examen físico apropiado, como pacientes inconscientes o bajo sedación. Inicialmente se propuso un valor igual o superior a $30 \mathrm{~mm} / \mathrm{Hg}$ como el límite para el diagnóstico del SCA, sin embargo, la presión de perfusión tisular o presión delta, también conocida como delta $\mathrm{P}$, la cual se obtiene restando la presión del compartimiento a la presión arterial diastólica, se ha asociado a un diagnóstico más preciso cuando el resultado es menor de $30 \mathrm{~mm} / \mathrm{Hg}$, y es de especial utilidad en el paciente politraumatizado en quien, como se explicó anteriormente, el SCA puede ocurrir con presiones sistémicas menores 7,17 .

La medición objetiva de la presión intracompartimental se realiza de manera invasiva y no invasiva. La técnica invasiva clásica se describió hacia 1975 y utiliza un manómetro de mercurio conectado a una columna de agua y a una aguja que se inserta en el compartimiento comprometido. Sin embargo, hoy existen distintos dispositivos comerciales que simplifican el procedimiento al hacer uso de un transductor digital; el más usado es el instrumento Stryker para monitoreo rápido de presión. Las recomendaciones actuales indican que, de existir fractura, la aguja debe colocarse en un radio de $5 \mathrm{~cm}$ adyacente a la misma y debe obtenerse registro de la presión de los 4 compartimientos ${ }^{4,6,15}$.

Las técnicas no invasivas pueden tener mayor utilidad en la población pediátrica, en la que la medición invasiva puede ser complicada, aunque se ha encontrado que el rendimiento de estas técnicas se ve significativamente afectado por la hipotensión sistémica ${ }^{4}$. Incluyen el uso de dispositivos de espectroscopia casi infrarroja, que mide la variación de oxígeno en el tejido muscular, y de ultrasonido.

La resonancia magnética tiene un rol limitado en el SCA ya que muestra edema y aumento del 
tamaño del compartimiento, que generalmente son cambios tardíos, por lo que tenerlos en cuenta puede retrasar el tratamiento oportuno.

Por último, para guiar el diagnóstico y vigilar la evolución de la enfermedad, se utilizan biomarcadores como la creatina quinasa (CK), mioglobinuria, recuento leucocitario (leucocitosis) y función renal, sin embargo, no existe un marcador especifico de isquemia muscular ${ }^{15}$. Un valor de CK mayor de $1000 \mathrm{mg} / \mathrm{dl}$ o la presencia de mioglobinuria son sugestivos de SCA y los niveles de CK continúan aumentando paulatinamente durante el curso de la enfermedad. Puede presentarse rabdomiólisis hasta en el $40 \%$ de los pacientes con SCA, secundario a traumatismo de la extremidad, y en presencia de esta puede haber deterioro de la función renal, con hipercalemia asociada ${ }^{26}$.

\section{Manejo}

El paciente con sospecha de SCA del miembro inferior debe ser valorado inmediatamente por un equipo quirúrgico. El abordaje inicial incluye la elevación del miembro afectado por encima del nivel del corazón y la remoción de vendajes compresivos, esto por sí solo puede reducir la presión intracompartimental en un $65-85 \%{ }^{4,25}$. Es importante además reducir las fracturas desplazadas, ya que así disminuye el edema asociado ${ }^{19}$. El manejo analgésico adecuado es clave para una correcta monitorización y seguimiento clínico del paciente, sin embargo se desaconseja el uso de bloqueos regionales que pueden interferir con el seguimiento oportuno ${ }^{24}$. Debido a que los pacientes hipotensos son más susceptibles a desarrollar el síndrome, la restauración del volumen circulante es clave para mantener presiones de perfusión adecuadas ${ }^{4}$.

La fasciotomía descompresiva es el único tratamiento efectivo una vez instaurado el SCA del miembro inferior ${ }^{7,15,22}$. Es un procedimiento quirúrgico que implica la realización de una o más incisiones en la piel y las fascias que separan los músculos en compartimientos aislados, permitiendo una descompresión mecánica de los mismos y la restauración de las presiones fisiológicas ${ }^{2}$. Las indicaciones incluyen la presencia de signos y sínto- mas sugestivos de SCA, una medición de la presión compartimental $>30 \mathrm{mmHg}$ o $>20 \mathrm{mmHg}$ en el paciente hipotenso y/o un delta de $\mathrm{P}<30 \mathrm{mmHg}^{1,9,15}$.

La fasciotomía profiláctica implica la realización del procedimiento previo a la instauración del SCA en un paciente cuyas condiciones plantean alto riesgo de desarrollarlo, por eso se indica en el contexto de lesión de vasos poplíteos, lesión arterial y venosa combinada, isquemia prolongada mayor de 6 horas, lesión por aplastamiento o lesión extensa de tejidos blandos asociada a fractura ${ }^{27}$. La principal contraindicación para la realización de fasciotomía es el diagnóstico tardío. Cuando el procedimiento se realiza 12 a 72 horas posterior a la instauración del SCA, se asocia a riesgo elevado de infección, amputación, falla renal por reperfusión de tejido muscular necrótico y altas tasas de mortalidad ${ }^{3}$.

Se entiende por fasciotomía temprana a aquella que se realiza en un periodo de 6 - 8 horas luego de la instauración del síndrome compartimental ${ }^{27,28}$. Múltiples autores han demostrado que un tiempo menor entre la instauración del SCA y la realización de la fasciotomía condiciona un mejor resultado funcional, menor tiempo de estancia hospitalaria y una menor tasa de complicaciones, incluyendo lesión muscular, lesión nerviosa y muerte ${ }^{19,27,28}$. Otro estudio demostró que la fasciotomía tardía, o la reintervención por fasciotomías incompletas, incrementa en 4 veces el riesgo de muerte ${ }^{29}$. En pacientes con lesión vascular, en los que se tiene planeado un reparo quirúrgico, se recomienda realizar la fasciotomía antes del reparo vascular, debido a que el compartimiento isquémico probablemente se encuentre edematizado y, por lo tanto, puede haber resistencia al flujo sanguíneo luego de su restauración, favoreciendo la aparición temprana de trombosis ${ }^{27}$.

Del total de las fasciotomías, el $68 \%$ se realizan en la pierna y el 8,9\% en el muslo ${ }^{1,3,30}$. La correcta realización de la fasciotomía es de suma importancia, ya que un procedimiento incompleto contribuye a perpetuar el síndrome compartimental e incrementa las complicaciones y resultados adversos ${ }^{6,29}$. Las técnicas de fasciotomía de pierna han sido ampliamente descritas en la literatura e 
incluyen la fasciotomía de incisión lateral única (con o sin fibulectomía) y la fasciotomía de incisión doble (lateral y medial), que es la más recomendada y comúnmente usada, por permitir una correcta liberación de los 4 compartimientos de una manera técnicamente menos exigente (figura 1 y 4$)^{6}$. La fasciotomía de incisión única puede ser considerada en traumas en los que hay fractura de tibia asociada, ya que la incisión doble reduce el soporte de los tejidos blandos y por lo tanto, da inestabilidad de la fractura ${ }^{31}$.

La incisión lateral permite liberar los compartimientos anterior y lateral $y$, se debe realizar aproximadamente $1 \mathrm{~cm}$ por delante del borde anterior del peroné, desde $3 \mathrm{~cm}$ por debajo de la cabeza hasta $3 \mathrm{~cm}$ por encima del maléolo lateral. Se replica la incisión en el tejido celular subcutáneo hasta exponer la fascia, teniendo precaución de no lesionar la vena safena menor y el nervio peroneo superficial, y en la fascia se realiza una incisión, que se extiende de manera longitudinal con la tijera de tejidos, para liberal el compartimiento anterior. Posteriormente, se identifica el septo intramuscular por los vasos perforantes y se realiza también una incisión longitudinal para la liberación del compartimiento lateral.

La incisión medial se realiza desde aproximadamente 2 - $3 \mathrm{~cm}$ medial al borde posterior de la tibia, desde la tuberosidad tibial hasta el maléolo medio. Este abordaje permite liberar el compartimiento posterior profundo y posterior superficial. Nuevamente se incide la piel y el tejido celular subcutáneo, teniendo precaución de no lesionar la vena safena mayor, hasta exponer la fascia. La fasciotomía del compartimiento posterior superficial se realiza primero. Al compartimiento posterior profundo se accede rechazando el músculo soleo del borde posterior de la tibia. Un error particularmente común consiste en ingresar al plano entre el músculo gastrocnemio y soleo y asumir que se ha liberado el compartimiento posterior profundo, por lo que identificar correctamente la cara posterior de la tibia y un adecuado entendimiento de la anatomía son fundamentales. La identificación del paquete neurovascular tibial posterior confirma el adecuado acceso a este compartimiento ${ }^{1,6,15}$.
En lo referente al SCA del muslo, debido a que la incidencia no es tan alta comparado con el de la pierna, los datos sobre las ventajas de las diferentes técnicas, las tasas de complicaciones y la etiología son escasos ${ }^{3}$. Aunque hay técnicas de incisión doble (medial y lateral), la más frecuentemente usada implica un abordaje lateral único (86\%), que discurre desde la línea intertrocantérica hasta el epicóndilo lateral del fémur, desde donde se descomprimen la totalidad de los compartimientos, lo cual se recomienda de manera rutinaria (figuras 2 y 5 ) $^{1}$.

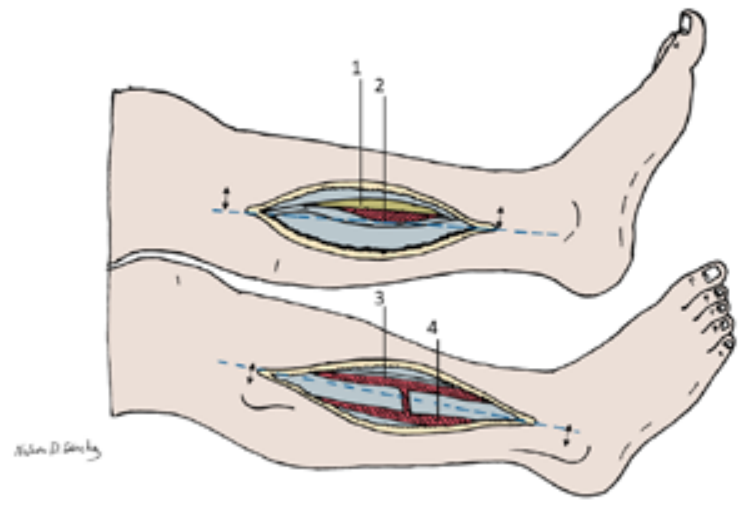

Figura 4. Fasciotomía de la pierna: Se muestra el abordaje medial (1-2) y lateral (3-4) de la pierna. 1. Tibia. 2. El compartimiento posterior profundo en estrecho contacto con la Tibia. 3. Fasciotomía del compartimiento anterior. 4. Fasciotomía del compartimiento lateral.

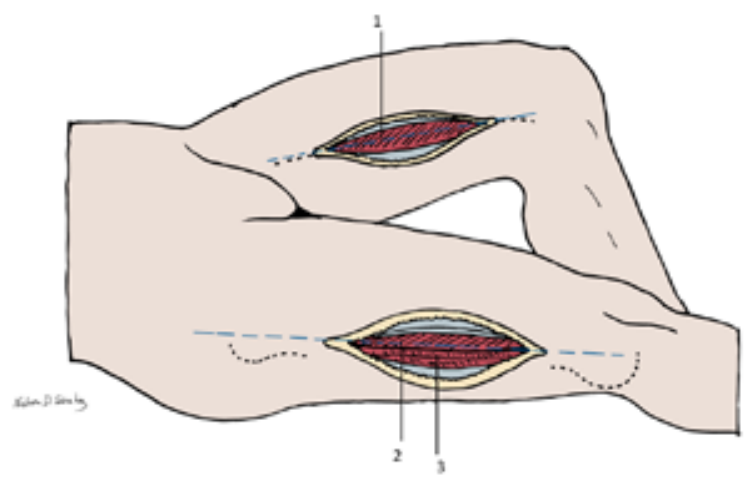

Figura 5. Fasciotomía del muslo: se muestra el abordaje medial (1) y el abordaje lateral del muslo (2-3). 1. Compartimiento medial. 2. Compartimiento anterior. 3. Compartimiento posterior. 
Se incide la piel y el tejido celular subcutáneo exponiendo el tracto iliotibial, sobre el cual se realiza una incisión, dividiendo luego la fascia del vasto lateral, para descomprimir el compartimiento anterior. Desde este punto se puede acceder al compartimiento posterior, que contiene los músculos isquiotibiales, retrayendo el vasto lateral y cortando sobre el septo intermuscular lateral. Los músculos aductores se agrupan en el compartimiento medial, al que se accede a continuación al incidir longitudinalmente sobre su fascia. Alternativamente este compartimiento puede liberarse desde una incisión longitudinal en la cara anteromedial del muslo de al menos 20 $\mathrm{cm}$, mediante la cual se expone e incide la fascia de los aductores o el septo intermuscular medial para liberar la presión ${ }^{15}$. El tejido muscular necrosado debe desbridarse durante la primera intervención y el paciente generalmente regresa al quirófano entre 24 a 72 horas para reevaluar la viabilidad muscular, recambio de apósitos y el cierre gradual de la herida ${ }^{6}$.

Una vez se regula la presión y se controlan los factores que desencadenaron el SCA, la herida debe cerrarse lo más pronto posible para evitar la retracción severa de la piel y las fascias ${ }^{26}$. Las técnicas de cierre incluyen el cierre primario, el cierre por segunda intención y el cierre primario tardío, sin que exista actualmente un consenso en cuanto a la mejor aproximación, por lo que la selección depende en gran medida del criterio y la experiencia del cirujano ${ }^{26}$. El cierre primario tardío puede lograrse entre los primeros 7 - 10 días, tiene los mejores resultados estéticos, funcionales y menor morbilidad. Sin embargo, con frecuencia no es factible debido al gran edema muscular, que ocasiona una protrusión importante de la masa muscular por la fascia y cierto grado de retracción cutánea. Se debe considerar además que el cierre primario prematuro puede desencadenar nuevamente un SCA y la reaproximación con demasiada tensión puede ocasionar necrosis de los bordes de la piel ${ }^{31}$.

El cierre por segunda intención solía ser otra técnica ampliamente usada, que ha caído en el abandono debido al elevado riesgo de infección, el tiempo prolongado de hospitalización, demora en el proceso de rehabilitación y pobres resultados estéticos ${ }^{32}$.
A la fecha, las técnicas mayormente utilizadas incluyen el cierre primario tardío asistido por sistemas de presión negativa, como el sistema $\mathrm{Va}$ cuum-Assited Clossure, que estimula la granulación, o por técnicas de dermotracción dinámica, como la del "cordón de zapato", que permiten una aproximación paulatina de los bordes de la herida y se pueden complementar con el uso de injertos de grosor parcial de piel de no lograrse el afrontamiento completo ${ }^{26,31,32}$.

\section{Complicaciones}

Las complicaciones de la fasciotomía no son infrecuentes. Una descompresión inadecuada de los compartimientos es, comúnmente, el error crítico que puede llevar a daño muscular y nervioso irreversible ${ }^{6}$. La rabdomiólisis ocurre hasta en el $42 \%$ de pacientes con SCA, secundaria a necrosis muscular, con daño y fallo renal agudo asociado a la liberación de mioglobina y otras citoquinas proinflamatorias al torrente sanguíneo; estas últimas además, contribuyen al aumento de las presiones intracompartimentales y el empeoramiento del cuadro ${ }^{33}$.

Aproximadamente un tercio de los pacientes presentará alguna complicación posoperatoria, las cuales incluyen la necrosis de tejidos blandos, dehiscencia de la herida luego del cierre, complicaciones relacionadas con los injertos, infección del sitio operatorio y retracción cutánea que complica el cierre ${ }^{28}$. La mortalidad global de los pacientes sometidos a fasciotomía se ubica alrededor del 11 - $15 \%$ y la amputación posterior a la misma se encuentra entre el $11-21 \%{ }^{1,3}$.

Velmahos et al. estudiaron las complicaciones en fasciotomias por SCA en trauma de extremidades ${ }^{28}$ durante un periodo de 4 años en un centro de trauma de alta complejidad y recolectaron información de 94 casos. El 31 \% de los pacientes desarrollaron complicaciones y el riesgo fue mayor en pacientes sometidos a fasciotomía de miembro inferior (34,3\% vs 20,8 \%), fasciotomía profiláctica versus terapéutica ( $42 \%$ vs $24,6 \%$ ), procedimiento realizado después de 8 horas (37\% vs $25 \%$ ) y heridas vasculares vs musculoesqueléticas ( $28,8 \%$ vs $22,5 \%$ ). Otros estudios apoyan el hallazgo de que la presencia de trauma vascular aumenta de manera significativa la tasa 
de complicaciones frente a aquellos pacientes que no presentan este tipo de lesiones ${ }^{27}$.

Considerando las importantes implicaciones médicas, funcionales, legales y psicológicas, la pérdida de la extremidad afectada, asociada al diagnóstico tardío, el tratamiento inadecuado o la etiología del SCA, es la complicación más grave 2,5. En los Estados Unidos se realizan anualmente más de 100.000 amputaciones del miembro inferior, incluyendo aquellas por encima y por debajo de la rodilla, y el cuidado de estos pacientes se extiende más allá de su hospitalización, ya que requieren rehabilitación intensa, adecuación de prótesis, optimización nutricional y una red de apoyo social significativa. Esto implica un alto costo a los sistemas de salud y, en general, una alta carga de enfermedad ${ }^{34}$.

Feliciano et al. reportaron una serie de pacientes llevados a amputación luego de desarrollar síndrome compartimental, identificando que el $75 \%$ se asociaba a retraso en recibir tratamiento adecuado ${ }^{2}$. Otros estudios han mostrado resultados similares. En una serie de fasciotomías en heridos en combate se encontró que los pacientes sometidos a fasciotomía temprana tuvieron $50 \%$ menos tasa de amputación que aquellos llevados a fasciotomía tardía ${ }^{29}$.

Por último, hay características propias del trauma de la extremidad que también predicen un pobre pronóstico o la necesidad de amputación temprana. Las fracturas abiertas Gustilo y Anderson tipo IIIB y IIIC tienen peor desenlace y las heridas severas de tejidos blandos, con avulsión y pérdida del tejido, constituyen la variable más importante a la hora de influenciar la decisión de amputar tempranamente, e incrementan de manera significativa el riesgo de complicaciones (infección crónica, dolor, falla de anastomosis vascular) ${ }^{19}$.

\section{Conclusiones}

Este artículo surge de una revisión amplia del conocimiento actual del síndrome compartimental agudo del miembro inferior. Un entendimiento adecuado de la anatomía y los procesos fisiopatológicos detrás del desarrollo de la enfermedad, permitirán al clínico el reconocimiento precoz de los signos y síntomas clásicos, para poder plantear un manejo oportuno y lograr su resolución. La fasciotomía es el manejo definitivo una vez instaurado el proceso mórbido y es altamente eficiente si se realiza de manera temprana y adecuada. Deben siempre descomprimirse la totalidad de los compartimientos, tanto en la pierna como en el muslo. El cirujano está llamado a revisar concienzudamente la técnica quirúrgica para evitar errores que puedan incrementar la morbimortalidad del paciente. La amputación se constituye como la complicación con mayor carga de enfermedad y se asocia mayormente a un diagnostico tardío o un manejo inadecuado del SCA.

Agradecimientos: Al siempre atento personal del Hospital Universitario Hernando Moncaleano Perdomo.

\section{Consideraciones éticas}

Consentimiento informado: Este estudio es una revisión de la literatura, y como tal no hay necesidad de un consentimiento informado ni de aprobación del Comité de Ética Institucional.

Conflicto de interés: ninguno declarado por los autores.

Financiación: Este trabajo fue financiado por los autores.

Contribuciones a los autores: Concepción y diseño del estudio, Análisis e interpretación de datos, redacción del manuscrito: David Felipe Muñoz-Pérez.

Concepción y diseño del estudio, revisión crítica y aprobación final del manuscrito: Rolando Medina-Rojas, Juan Felipe Sanjuan-Marín, Roberto José Rodríguez-Flórez.

Redacción del manuscrito y producción original de las ilustraciones: Nelson Daniel Sánchez-Arroyo.

Los autores declaramos que el manuscrito ha sido leído en su totalidad y aprobado por todos lo que nos permite asumir la responsabilidad pública del contenido del mismo según las prácticas COPE.

\section{Referencias}

1. Long B, Koyfman A, Gottlieb M. Evaluation and management of acute compartment syndrome in the emergency department. J Emerg Med. 2019;56:386-97. https://doi.org/10.1016/j.jemermed.2018.12.021 
2. Feliciano DV, Cruse PA, Spjut-Patrinely V, Burch JM, Mattox KL. Fasciotomy after trauma to the extremities. Am J Surg. 1988;156:533-6. https://doi.org/10.1016/S0002-9610(88)80547-6

3. Branco BC, Inaba K, Barmparas G, Schnüriger B, Lustenberger T, Talving $\mathrm{P}$, et al. Incidence and predictors for the need for fasciotomy after extremity trauma: A 10 -year review in a mature level I trauma centre. Injury. 2011;42:1157-63. https://doi.org/10.1016/j.injury.2010.07.243

4. Duckworth AD, McQueen MM. The diagnosis of acute compartment syndrome : A critical analysis review. JBJS Rev. 2017;5:e1. https://doi.org/10.2106/JBJS.RVW.17.00016

5. Heemskerk J, Kitslaar P. Acute compartment syndrome of the lower leg: Retrospective study on prevalence, technique, and outcome of fasciotomies. World J Surg. 2003;27:744-7. https://doi.org/10.1007/s00268-003-6691-7

6. Cone J, Inaba K. Lower extremity compartment syndrome. Trauma Surg Acute Care Open. 2017;2:1-6. https://doi.org/10.1136/tsaco-2017-000094

7. Garner MR, Taylor SA, Gausden E, Lyden JP. Compartment syndrome: diagnosis, management, and unique concerns in the twenty-first century. HSS J. 2014;10:143-52.

https://doi.org/10.1007/s11420-014-9386-8

8. Abouezzi Z, Nassoura Z, Ivatury RR, Porter JM, Stahl WM. A critical reappraisal of indications for fasciotomy after extremity vascular trauma. Arch Surg. 1998;133:54751. https://doi.org/10.1001/archsurg.133.5.547

9. Swain R, Ross D. Lower extremity compartment syndrome. When to suspect acute or chronic pressure buildup. Postgrad Med. 2009;105:159-68. https://doi.org/10.3810/pgm.1999.03.599

10. Carter AB, Richards RL, Zachary RB. The anterior tibial syndrome. Lancet. 1949; (case 1):730-6.

11. Bywaters EGL, Beall D. Crush injuries with impairment of renal function. Br Med J. 1941;1(4185):427. https://doi.org/10.1136/bmj.1.4185.427

12. Gonzalez RP, Scott W, Wright A, Phelan HA, Rodning $\mathrm{CB}$. Anatomic location of penetrating lower-extremity trauma predicts compartment syndrome development. Am J Surg. 2009;197:371-5. https://doi.org/10.1016/j.amjsurg.2008.11.013

13. Frink M, Hildebrand F, Krettek C, Brand J, Hankemeier S. Compartment syndrome of the lower leg and foot. Clin Orthop Relat Res. 2010;468:940-50. https://doi.org/10.1007/s11999-009-0891-x

14. Olson SA, Glasgow RR. Acute compartment syndrome in lower extremity musculoskeletal trauma. J Am Acad Orthop Surg. 2005;13:436-44. https://doi.org/10.5435/00124635-200511000-00003
15. Mabvuure NT, Malahias M, Hindocha S, Khan W, Juma A. Acute compartment syndrome of the limbs: current concepts and management. Open Orthop J. 2012;6:53543. https://doi.org/10.2174/1874325001206010535

16. Gourgiotis S, Villias C, Germanos S, Foukas A, Ridolfini MP. Acute limb compartment syndrome: A review. J Surg Educ. 2007;64:178-86. https://doi.org/10.1016/j.jsurg.2007.03.006

17. McQueen MM, Duckworth AD. The diagnosis of acute compartment syndrome: a review. Eur J Trauma Emerg Surg. 2014;40:521-8. https://doi.org/10.1007/s00068-014-0414-7

18. McQueen MM, Duckworth AD, Aitken SA, Sharma RA, Court-Brown CM. Predictors of compartment syndrome after tibial fracture. J Orthop Trauma. 2015;29:451-5. https://doi.org/10.1097/BOT.0000000000000347

19. McQueen MM, Christie J, Court-Brown CM. Acute compartment syndrome in tibial diaphyseal fractures. J Bone Jt Surg. 1996;78:95-8. https://doi.org/10.1302/0301-620X.78B1.0780095

20. Mauser N, Gissel H, Henderson C, Hao J, Hak D, Mauffrey C. Acute lower-leg compartment syndrome. Orthopedics. 2013;36:619-24. https://doi.org/10.3928/01477447-20130724-07

21. Tremblay LN, Feliciano DV, Rozycki GS. Secondary extremity compartment syndrome. J Trauma. 2002;53: 833-7.

https://doi.org/10.1097/00005373-200211000-00005

22. McQueen MM, Gaston P, Court-Brown CM. Acute compartment syndrome. Who is at risk? J Bone Joint Surg Br. 2000;82:200-3. https://doi.org/10.1302/0301-620X.82B2.9799

23. Pechar J, Lyons MM. Acute compartment syndrome of the lower leg: A review. J Nurse Pract. 2016;12:265-70. https://doi.org/10.1016/j.nurpra.2015.10.013

24. Badhe S, Baiju D, Elliot R, Rowles J, Calthorpe D. The 'silent' compartment syndrome. Injury. 2009;40:220-2. https://doi.org/10.1016/j.injury.2008.07.023

25. Ulmer $\mathrm{T}$. The clinical diagnosis of compartment syndrome of the lower leg: Are clinical findings predictive of the disorder? J Orthop Trauma. 2002;16:572-7. https://doi.org/10.1097/00005131-200209000-00006

26. Jauregui JJ, Yarmis SJ, Tsai J, Onuoha KO, Illical E, Paulino CB. Fasciotomy closure techniques. J Orthop Surg. 2017;25:230949901668472. https://doi.org/10.1177/2309499016684724

27. Farber A, Tan TW, Hamburg NM, Kalish JA, Joglar F, Onigman T, et al. Early fasciotomy in patients with extremity vascular injury is associated with decreased risk of adverse limb outcomes: A review of the National Trauma Data Bank. Injury. 2012;43:1486-91. https://doi.org/10.1016/j.injury.2011.06.006 
28. Velmahos GC, Theodorou D, Demetriades D, Chan L, Berne TV, Asensio J, et al. Complications and nonclosure rates of fasciotomy for trauma and related risk factors. World J Surg. 1997;21:247-53.

https://doi.org/10.1007/s002689900224

29. Ritenour AE, Dorlac WC, Fang R, Woods T, Jenkins DH, Flaherty SF, et al. Complications after fasciotomy revision and delayed compartment release in combat patients. J Trauma. 2008;64(2 Suppl):S153-62. https://doi.org/10.1097/TA.0b013e3181607750

30. Hoyos-Gomez JC, Morales CH. Fasciotomía profiláctica y síndrome "compartimental " de las extremidades: ¿existen indicaciones justificables? Rev Colomb Cir. 2011;26:101-10.

31. Kakagia D, Karadimas EJ, Drosos G, Ververidis A, Trypsiannis G, Verettas D. Wound closure of leg fasciotomy:
Comparison of vacuum-assisted closure versus shoelace technique. A randomised study. Injury. 2014;45:890-3. https://doi.org/10.1016/j.injury.2012.02.002

32. Weaver MJ, Owen TM, Morgan JH, Harris MB. Delayed primary closure of fasciotomy incisions in the lower leg. J Orthop Trauma. 2015;29:308-11. https://doi.org/10.1097/BOT.0000000000000278

33. Tsai WH, Huang ST, Liu WC, Chen LW, Yang KC, Hsu KC, et al. High risk of rhabdomyolysis and acute kidney injury after traumatic limb compartment syndrome. Ann Plast Surg. 2015;74:S158-61. https://doi.org/10.1097/SAP.0000000000000460

34. Wise ES, McMaster WG, Williamson K, Wergin JE, Hokking KM, Brophy CM. Preoperative predictors of 30-day mortality and prolonged length of stay after above-knee amputation. Ann Vasc Surg. 2016;31:124-33.

http://dx.doi.org/10.1016/j.avsg.2015.08.017 